\title{
The explosive life of massive binaries
}

\author{
M. Renzo ${ }^{1,2}$ and E. Zapartas ${ }^{3}$ \\ ${ }^{1}$ Center for Computational Astrophysics, Flatiron Institute, New York, NY \\ 10010, USA \\ 2 Anton Pannekoek Institute for Astronomy and Grappa, University of \\ Amsterdam, NL-1090 GE Amsterdam, The Netherlands \\ 3 Geneva Observatory, University of Geneva, CH-1290 Sauverny, Switzerland
}

Received: December 2, 2019; Accepted: January 26, 2020

\begin{abstract}
Massive stars are born predominantly as members of binary (or higher multiplicity) systems, and the presence of a companion can significantly alter their life and final fate. Therefore, any observed sample of massive stars or associated transients is likely to be significantly influenced by the effects of binarity. Here, we focus on the relationship between massive binary evolution and core-collapse supernova events. In the vast majority of the cases, the first core-collapse event happening in a binary system unbinds the two stars. Studying the population of companion stars, either at the supernova site, or as "widowed" stars long after the explosion, can be used to constrain the previous orbital evolution of the binary progenitor, and explosion physics of their former companion. Specifically, the population of "widowed" stars might provide statistical constraints on the typical amplitude of black hole natal kicks without seeing neither the black holes nor the transient possibly associated to their formation. Binarity also has a large impact on the predicted population of supernova sub-types, including hydrogen-rich type II supernovae, with a significant fraction of hydrogen-rich stars at explosions being either merger products or accretors.
\end{abstract}

Key words: stars: massive - binaries - supernovae

\section{Massive stars and binarity}

A variety of observations suggest that the vast majority of massive stars are born in binary (e.g., Mason et al., 2009; Sana \& Evans, 2011; Almeida et al., 2017) or higher multiplicity systems (e.g., Tokovinin, 2008), and that up to $\sim 70 \%$ of the O-type stars might exchange mass or merge with a companion before the end of their evolution (e.g., Sana et al., 2012).

This implies that observational samples of massive stars and/or transients is likely to contain binary evolution products (e.g., Langer 2012 for a review, and de Mink et al. 2014).

In the era of large surveys, such as Gaia (Gaia Collaboration et al., 2018) or ZTF (Bellm, 2014) and LSST (LSST Science Collaboration et al., 2009) in the 
time domain, we have the opportunity to investigate both the most common and the rare and exotic binary evolution paths.

Here, we focus on some aspects of the relationship between binarity and core-collapse supernova (CCSN) explosions. We refer interested readers for more details to our studies in Renzo et al. (2019); Zapartas et al. (2017a,b, 2019). We describe the population synthesis approach in Section 1.1. In Section 2, we focus on the consequences of the first CCSN explosion for the binary system, while in Section 3 we discuss the implications of binary evolution of the progenitors for the population of CCSN events.

\subsection{Population synthesis}

The evolution of a star is determined mainly by its initial mass, and secondly by its rotation rate and metallicity ${ }^{1}(Z)$. Nevertheless, many uncertain or unknown parameters enter in the modeling of internal processes in stars (poorly known nuclear reaction rates, modeling of mixing processes, wind mass loss rates, etc.). Exploring the parameter space for single star evolution is a challenging task still being actively pursued (e.g., Woosley, 2017; Renzo et al., 2017; Sukhbold et al., 2018; Woosley, 2019; Farmer et al., 2019, for recent studies of single massive stars). When considering the evolution of two stars born together in a binary, i.e., the standard for massive stars, the number of dimensions of the parameter space increases very rapidly: not only the evolution of a massive binary system depends on the two initial masses of the stars, but also their initial orbital period and, possibly, eccentricity. Moreover, the number of free or poorly constrained parameters entering in the models also increases (e.g., stability and efficiency of mass transfer, angular momentum losses, treatment of common envelope), reflecting the current insufficient understanding of the physics of binary interactions.

The complexity of the problem and the vastness of the parameter space to explore require to resort to population synthesis techniques, i.e., broadly speaking, building synthetic populations by weighting with initial distributions pre-computed models. This can be done with grids of detailed binary evolution models (e.g., Justham et al., 2014; Marchant et al., 2016; Eldridge et al., 2017), which have the advantage of solving the differential equations describing the evolution and interaction of the two stars, but are limited to the values of unknown parameters for which the computation is numerically feasible.

The common alternative is to rely on pre-computed single star models (e.g., Pols et al., 1998; Hurley et al., 2000) paired with analytic algorithms to represent the binary interactions (e.g., Tout et al., 1997; Hurley et al., 2002), which allow for the exploration of larger portions of the parameter space of binary evolution at the cost of a reduced physical accuracy of the models, and thus limited predictive power of a single population. The results we present here

${ }^{1}$ Specifically, its initial iron content, e.g., Tramper et al. 2016. 
were obtained with this approach, using the binary_c code (Izzard et al., 2004, 2006, 2009; Izzard et al., 2018).

The speed of these simulations (typically $\lesssim 0.1 \mathrm{sec}$ per binary) allows for reruns varying the uncertain parameters. If a particular result is found to survive all the parameter variations possible, the prediction can be considered robust. Vice versa, if a result is found to be sensitive to variations of a particular unknown parameter, the comparison with observed populations has the potential of constraining such parameter. The ranges reported below were obtained with one-by-one variations of uncertain parameters ${ }^{2}$ in the models to assess the robustness of the predictions made.

\section{How explosions can affect binaries}

While the majority of massive stars are born with companion(s), only a small minority remain bound after the first core-collapse in the system (e.g. De Donder et al., 1997; Eldridge et al., 2011; Renzo et al., 2019). For compact objects (i.e., neutron stars and black holes), being in a binary system is the exception rather than the rule. This is counterintuitive, since binary interactions are often the main or only way to observe the compact objects (e.g., through X-rays and/or gravitational waves), especially in the case of black holes. In other words, the majority of (isolated) massive binaries evolves to form a single compact object and a "widowed" companion star.

The main reason why $86_{-22}^{+11} \%$ of binaries ${ }^{3}$ are disrupted at the first corecollapse event appears to be supernova (SN) natal kicks (Renzo et al., 2019). The width of the range reported above is dominated by the uncertainties in the parametrization of the natal kicks, which allows to use the population of "widowed stars" to observationally constrain the explosion physics of their former companions in a statistical sense. In particular, the high-mass tail of the mass function of "widowed stars" is sensitive to the average black hole kick (Renzo et al., 2019): black holes are produced by on average more massive stars, which typically have more massive companion that can become single "widowed stars" if the black holes receive significant kicks at formation.

When assuming spherical symmetry of the collapse and explosion in the frame of the exploding star (i.e., no natal kick), only $\sim 16 \%$ of binary systems are disrupted. In this cases, the change in gravitational potential due to the rapid loss of the SN ejecta from the binary (the so-called "Blaauw kick," Blaauw 1961) alone unbinds the binary (see also Boubert et al. 2017). However, typically Roche-lobe overflow will strip the envelope of the donor star which explodes first, limiting the amount of mass that can be ejected at explosion. Only for

${ }^{2}$ Note however that this approach neglects possible physical correlations between the parameters, see also, e.g., Andrews et al. 2018; Taylor \& Gerosa 2018.

${ }^{3}$ This fraction excludes binaries that result in a stellar merger before the first CCSN. 
wide, non-interacting binaries the "Blaauw kick" is sufficient to separate the companions.

If the core-collapse produces a successful explosion, the newly "widowed star" will be hit by the blast wave (e.g., Moriya et al., 2015; Hirai et al., 2018), which can alter its appearance for a few thermal timescales by depositing energy in the star and removing some mass, although the latter effect is typically small (e.g. Liu et al., 2015; Rimoldi et al., 2016).

Because of the binary disruption, the "widowed star" acquires a peculiar space velocity corresponding to first order to its pre-explosion orbital velocity. Occasionally, this can produce fast moving runaway stars (e.g., Blaauw, 1961; Hoogerwerf et al., 2001), however, it is much more common that this peculiar velocity is relatively slow $\left(\sim 10 \mathrm{~km} \mathrm{~s}^{-1}\right)$, making them "walkaway stars" (Renzo et al., 2019). This happens because during the first stable Roche lobe overflow, long before the SN explosion, binaries tend to overall widen. Moreover, the mass transfer leads to an inversion of the mass ratio. Both effects decrease the orbital velocity of the secondary. The velocity distribution of "widowed stars," if observed, would add a constraint on the orbital evolution of massive binaries: their peculiar velocity with respect to their parent population relates to how close to the companion they were at the time of the explosion.

\section{How binaries can affect the explosions}

Binarity can have consequences on the rate and timing of CCSN events (e.g., Podsiadlowski et al., 1992; De Donder \& Vanbeveren, 2003; Zapartas et al., 2017a). For example, it can allow stars born below the minimum mass to give a CCSN to ultimately explode (either because they accreted mass from a companion, or because of mergers). Zapartas et al. (2017a) showed that $15_{-8}^{+9} \%$ of all CCSNe might come from this type of evolutionary paths. These progenitor systems are generally longer-lived than normal massive stars, resulting in delayed SNe compared to the age of a given (co-eval) parent population. More convoluted evolutionary paths involving multiple phases of mass transfer (either stable or unstable) might also possibly generate non-standard pre-explosion stellar structures resulting in peculiar transients (e.g., Justham et al., 2014; Menon \& Heger, 2017).

\subsection{Stripped-envelope $\mathrm{SNe}$}

Binarity also impacts the observable properties of the stellar explosions themselves: as mentioned above, the most common binary evolution path involves a phase of stable mass transfer which typically removes the entire hydrogen-rich envelope of the donor star (e.g., Kippenhahn \& Weigert, 1967; Yoon et al., 2017; Götberg et al., 2017, although this is known to be Z-dependent). Therefore, typically the first SN in the system will be a hydrogen-less type Ib or Ic SN, or 
possibly a IIb with only a little amount of hydrogen remaining, commonly referred to all together as stripped-envelope SNe (e.g., Smith et al., 2011; Eldridge et al., 2013).

Zapartas et al. (2017b) showed that about 2/3 of all stripped-envelope $\mathrm{SNe}$ are expected to occur in the presence of a main sequence companion, assuming an initial mixture of single and binary stars and sub-solar Z. This fraction drops below $1 / 2$ only for parameter variations enhancing the wind mass loss, including super-solar Z. These SNe are those unbinding the binary and creating the "widowed" stars. Albeit common, the main sequence companions might be challenging to find at the SN site because of their possibly low mass (Zapartas et al., 2017b).

Most of the stripped-envelope SNe progenitors single at explosion were massive enough to get stripped through winds, either because they were initially massive enough or because they accreted mass or merged with a binary companion. In $5_{-4}^{+12 \%}$ of the cases, the exploding star had a compact object companion.

\subsection{H-rich $\mathrm{SNe}$}

Perhaps more surprisingly, binary products might also contribute to a significant fraction of hydrogen-rich SNe, despite the fact these can in principle be explained by single star evolution. Single star models struggle to explain the variety of light curve morphology and spectral evolution of these hydrogen-rich explosions. Using observationally motivated initial distributions, Zapartas et al. (2019) showed that accretion, and more importantly mergers in binaries can lead to stars exploding with a significant amount of hydrogen left in their envelope. These SNe with a binary-product progenitor could contribute to $45_{-12}^{+8} \%$ of all hydrogen-rich SNe (see also Eldridge et al. 2019). The "widowed" stars alone can contribute to $14_{-11}^{+4} \%$ of hydrogen-rich SNe.

\section{Conclusions}

Massive binary evolution can proceed through a complex variety of paths depending on both initial conditions and physical assumptions of the models. While theoretical understanding is far from final, the exploration of the vast parameter space is becoming possible. Existing and upcoming observational constraints from large surveys and gravitational waves are already guiding it. Loosely speaking, neglecting stellar mergers, each massive binary will produce a close-to hydrogen-less donor star and a hydrogen-rich accreting star. The outcome of stellar mergers depends on the evolutionary phases of the two stars when they happen. Accounting for binarity when dealing with observed samples of massive stars (even if presently single) and samples of CCSNe is important to not misinterpret the observations. 
Acknowledgements. We are grateful for guidance and mentoring to S. E. de Mink, and to R. G. Izzard for developing and maintaining the binary_c code, and giving us access and support. We have benefitted from uncountable enlightening discussions with F. Broekgaarden, R. Farmer, Y. Götberg, D. Hendricks, S. Justham, E. Laplace, N. Smith, K. Temmink, S. Toonen, A. van Son, D. Vartanyan. EZ acknowledges support from the Federal Commission for Scholarships for Foreign Students for the Swiss Government Excellence Scholarship (ESKAS No. 2019.0091) for the academic year 2019-2020

\section{References}

Almeida, L. A., Sana, H., Taylor, W., et al., The Tarantula Massive Binary Monitoring. I. Observational campaign and OB-type spectroscopic binaries. 2017, Astron. Astrophys., 598, A84, DOI: 10.1051/0004-6361/201629844

Andrews, J. J., Zezas, A., \& Fragos, T., dart_board: Binary Population Synthesis with Markov Chain Monte Carlo. 2018, Astrophys. J., Suppl., 237, 1, DOI: 10.3847/15384365 /aaca30

Bellm, E., The Zwicky Transient Facility. 2014, in The Third Hot-wiring the Transient Universe Workshop, ed. P. R. Wozniak, M. J. Graham, A. A. Mahabal, \& R. Seaman, 27-33

Blaauw, A., On the origin of the O- and B-type stars with high velocities (the "runaway" stars), and some related problems. 1961, Bull. Astron. Inst. Netherlands, 15, 265

Boubert, D., Fraser, M., Evans, N. W., Green, D. A., \& Izzard, R. G., Binary companions of nearby supernova remnants found with Gaia. 2017, Astron. Astrophys., 606, A14, DOI: 10.1051/0004-6361/201731142

De Donder, E. \& Vanbeveren, D., The galactic evolution of the supernova rates. 2003, New Astronomy, 8, 817, DOI: 10.1016/S1384-1076(03)00069-1

De Donder, E., Vanbeveren, D., \& van Bever, J., The number of O-type runaways, the number of $\mathrm{O}$ and Wolf-Rayet stars with a compact companion and the formation rate of double pulsars predicted by massive close binary evolution. 1997, Astron. Astrophys., 318, 812

de Mink, S. E., Sana, H., Langer, N., Izzard, R. G., \& Schneider, F. R. N., The Incidence of Stellar Mergers and Mass Gainers among Massive Stars. 2014, Astrophys. J., 782, 7, DOI: 10.1088/0004-637X/782/1/7

Eldridge, J. J., Fraser, M., Smartt, S. J., Maund, J. R., \& Crockett, R. M., The death of massive stars - II. Observational constraints on the progenitors of Type Ibc supernovae. 2013, Mon. Not. R. Astron. Soc., 436, 774, DOI: 10.1093/mnras/stt1612

Eldridge, J. J., Guo, N. Y., Rodrigues, N., Stanway, E. R., \& Xiao, L., Supernova lightCURVE POPulation Synthesis II: Validation against supernovae with an observed progenitor. 2019, arXiv e-prints, arXiv:1908.07762

Eldridge, J. J., Langer, N., \& Tout, C. A., Runaway stars as progenitors of supernovae and gamma-ray bursts. 2011, Mon. Not. R. Astron. Soc., 414, 3501, DOI: 10.1111/j.1365-2966.2011.18650.x 
Eldridge, J. J., Stanway, E. R., Xiao, L., et al., Binary Population and Spectral Synthesis Version 2.1: Construction, Observational Verification, and New Results. 2017, PASA, 34, e058, DOI: 10.1017/pasa.2017.51

Farmer, R., Renzo, M., de Mink, S. E., Marchant, P., \& Justham, S., Mind the gap: The location of the lower edge of the pair instability supernovae black hole mass gap. 2019, arXiv e-prints, arXiv:1910.12874

Gaia Collaboration, Brown, A. G. A., Vallenari, A., et al., Gaia Data Release 2. Summary of the contents and survey properties. 2018, Astron. Astrophys., 616, A1, DOI: 10.1051/0004-6361/201833051

Götberg, Y., de Mink, S. E., \& Groh, J. H., Ionizing spectra of stars that lose their envelope through interaction with a binary companion: role of metallicity. 2017, Astron. Astrophys., 608, A11, DOI: 10.1051/0004-6361/201730472

Hirai, R., Podsiadlowski, P., \& Yamada, S., Comprehensive study of ejecta-companion interaction for core-collapse supernovae in massive binaries. 2018 [arXiv1803.10808]

Hoogerwerf, R., de Bruijne, J. H. J., \& de Zeeuw, P. T., On the origin of the O and B-type stars with high velocities. II. Runaway stars and pulsars ejected from the nearby young stellar groups. 2001, Astron. Astrophys., 365, 49, DOI: 10.1051/00046361:20000014

Hurley, J. R., Pols, O. R., \& Tout, C. A., Comprehensive analytic formulae for stellar evolution as a function of mass and metallicity. 2000, Mon. Not. R. Astron. Soc., 315, 543, DOI: $10.1046 /$ j.1365-8711.2000.03426.x

Hurley, J. R., Tout, C. A., \& Pols, O. R., Evolution of binary stars and the effect of tides on binary populations. 2002, Mon. Not. R. Astron. Soc., 329, 897, DOI: 10.1046/j.1365-8711.2002.05038.x

Izzard, R. G., Dray, L. M., Karakas, A. I., Lugaro, M., \& Tout, C. A., Population nucleosynthesis in single and binary stars. I. Model. 2006, Astron. Astrophys., 460, 565, DOI: 10.1051/0004-6361:20066129

Izzard, R. G., Glebbeek, E., Stancliffe, R. J., \& Pols, O. R., Population synthesis of binary carbon-enhanced metal-poor stars. 2009, Astron. Astrophys., 508, 1359, DOI: 10.1051/0004-6361/200912827

Izzard, R. G., Preece, H., Jofre, P., et al., Binary stars in the Galactic thick disc. 2018, Mon. Not. R. Astron. Soc., 473, 2984, DOI: 10.1093/mnras/stx2355

Izzard, R. G., Tout, C. A., Karakas, A. I., \& Pols, O. R., A new synthetic model for asymptotic giant branch stars. 2004, Mon. Not. R. Astron. Soc., 350, 407, DOI: 10.1111/j.1365-2966.2004.07446.x

Justham, S., Podsiadlowski, P., \& Vink, J. S., Luminous Blue Variables and Superluminous Supernovae from Binary Mergers. 2014, Astrophys. J., 796, 121, DOI: 10.1088/0004-637X/796/2/121

Kippenhahn, R. \& Weigert, A., Entwicklung in engen Doppelsternsystemen I. Massenaustausch vor und nach Beendigung des zentralen Wasserstoff-Brennens. 1967, $Z A p, 65,251$ 
Langer, N., Presupernova Evolution of Massive Single and Binary Stars. 2012, Ann. Rev. Astron. Astrophys., 50, 107

Liu, Z.-W., Tauris, T. M., Röpke, F. K., et al., The interaction of core-collapse supernova ejecta with a companion star. 2015, Astron. Astrophys., 584, A11, DOI: $10.1051 / 0004-6361 / 201526757$

LSST Science Collaboration, Abell, P. A., Allison, J., et al., LSST Science Book, Version 2.0. 2009 [arXiv0912.0201]

Marchant, P., Langer, N., Podsiadlowski, P., Tauris, T. M., \& Moriya, T. J., A new route towards merging massive black holes. 2016, Astron. Astrophys., 588, A50, DOI: $10.1051 / 0004-6361 / 201628133$

Mason, B. D., Hartkopf, W. I., Gies, D. R., Henry, T. J., \& Helsel, J. W., The High Angular Resolution Multiplicity of Massive Stars. 2009, Astron. J., 137, 3358, DOI: 10.1088/0004-6256/137/2/3358

Menon, A. \& Heger, A., The quest for blue supergiants: binary merger models for the evolution of the progenitor of SN 1987A. 2017, Mon. Not. R. Astron. Soc., 469, 4649

Moriya, T. J., Liu, Z.-W., \& Izzard, R. G., Observable fractions of core-collapse supernova light curves brightened by binary companions. 2015, Mon. Not. R. Astron. Soc., 450, 3264, DOI: 10.1093/mnras/stv934

Podsiadlowski, P., Joss, P. C., \& Hsu, J. J. L., Presupernova evolution in massive interacting binaries. 1992, Astrophys. J., 391, 246, DOI: 10.1086/171341

Pols, O. R., Schröder, K.-P., Hurley, J. R., Tout, C., \& Eggleton, P., Stellar evolution models for $\mathrm{Z}=0.0001$ to 0.03. 1998, Mon. Not. R. Astron. Soc., 298, 525, DOI: 10.1046/j.1365-8711.1998.01658.x

Renzo, M., Ott, C. D., Shore, S. N., \& de Mink, S. E., Systematic survey of the effects of wind mass loss algorithms on the evolution of single massive stars. 2017, Astron. Astrophys., 603, A118, DOI: 10.1051/0004-6361/201730698

Renzo, M., Zapartas, E., de Mink, S. E., et al., Massive runaway and walkaway stars. A study of the kinematical imprints of the physical processes governing the evolution and explosion of their binary progenitors. 2019, Astron. Astrophys., 624, A66, DOI: 10.1051/0004-6361/201833297

Rimoldi, A., Portegies Zwart, S., \& Rossi, E. M., Simulations of stripped corecollapse supernovae in close binaries. 2016, Comput. Astroph. and Cosm., 3, 2, DOI: $10.1186 / \mathrm{s} 40668-016-0015-4$

Sana, H., de Mink, S. E., de Koter, A., et al., Binary Interaction Dominates the Evolution of Massive Stars. 2012, Science, 337, 444, DOI: 10.1126/science.1223344

Sana, H. \& Evans, C. J., The multiplicity of massive stars. 2011, in IAU Symposium, Vol. 272, Active OB Stars: Structure, Evolution, Mass Loss, and Critical Limits, $474-485$

Smith, N., Li, W., Filippenko, A. V., \& Chornock, R., Observed fractions of corecollapse supernova types and initial masses of their single and binary progen- 
itor stars. 2011, Mon. Not. R. Astron. Soc., 412, 1522, DOI: 10.1111/j.13652966.2011.17229.x

Sukhbold, T., Woosley, S. E., \& Heger, A., A High-resolution Study of Presupernova Core Structure. 2018, Astrophys. J., 860, 93, DOI: 10.3847/1538-4357/aac2da

Taylor, S. R. \& Gerosa, D., Mining gravitational-wave catalogs to understand binary stellar evolution: A new hierarchical Bayesian framework. 2018, Phys. Rev. D, 98, 083017, DOI: 10.1103/PhysRevD.98.083017

Tokovinin, A., Comparative statistics and origin of triple and quadruple stars. 2008, Mon. Not. R. Astron. Soc., 389, 925, DOI: 10.1111/j.1365-2966.2008.13613.x

Tout, C. A., Aarseth, S. J., Pols, O. R., \& Eggleton, P. P., Rapid binary star evolution for N-body simulations and population synthesis. 1997, Mon. Not. R. Astron. Soc., 291, 732, DOI: $10.1093 /$ mnras/291.4.732

Tramper, F., Sana, H., \& de Koter, A., A New Prescription for the Mass-loss Rates of WC and WO Stars. 2016, Astrophys. J., 833, 133, DOI: 10.3847/1538$4357 / 833 / 2 / 133$

Woosley, S. E., Pulsational Pair-instability Supernovae. 2017, Astrophys. J., 836, 244 DOI: $10.3847 / 1538-4357 / 836 / 2 / 244$

Woosley, S. E., The Evolution of Massive Helium Stars, Including Mass Loss. 2019, Astrophys. J., 878, 49, DOI: 10.3847/1538-4357/ab1b41

Yoon, S.-C., Dessart, L., \& Clocchiatti, A., Type Ib and IIb Supernova Progenitors in Interacting Binary Systems. 2017, Astrophys. J., 840, 10, DOI: 10.3847/15384357/aa6afe

Zapartas, E., de Mink, S. E., Izzard, R. G., et al., Delay-time distribution of corecollapse supernovae with late events resulting from binary interaction. 2017a, Astron. Astrophys., 601, A29, DOI: 10.1051/0004-6361/201629685

Zapartas, E., de Mink, S. E., Justham, S., et al., The diverse lives of progenitors of hydrogen-rich core-collapse supernovae: the role of binary interaction. 2019 [arXiv1907.06687]

Zapartas, E., de Mink, S. E., Van Dyk, S. D., et al., Predicting the Presence of Companions for Stripped-envelope Supernovae: The Case of the Broad-lined Type Ic SN 2002ap. 2017b, Astrophys. J., 842, 125, DOI: 10.3847/1538-4357/aa7467 